Meta

Journal des traducteurs

Translators' Journal

\title{
La traduction de la Sophonisbe de Trissino par Melin de Saint-Gelais
}

\section{Pierre Van Rutten}

Volume 29, numéro 2, juin 1984

URI : https://id.erudit.org/iderudit/004554ar

DOI : https://doi.org/10.7202/004554ar

Aller au sommaire du numéro

Éditeur(s)

Les Presses de l'Université de Montréal

ISSN

0026-0452 (imprimé)

1492-1421 (numérique)

Découvrir la revue

Citer cette note

Van Rutten, P. (1984). La traduction de la Sophonisbe de Trissino par Melin de Saint-Gelais. Meta, 29(2), 224-226. https://doi.org/10.7202/004554ar d'utilisation que vous pouvez consulter en ligne.

https://apropos.erudit.org/fr/usagers/politique-dutilisation/ 
Linda Gaboriau ${ }^{15}$ ou le poème de Sylvia Plath "Three Women" traduit par Laure Vernière" ${ }^{16}$, toutes ces traductions montrent une recherche beaucoup plus systématique du mot concret, en particulier au niveau biophysiologique. Dans ses traductions des poèmes de Cécile Cloutier, Barbara Godard insiste plus que Alexandre Amprimoz sur le toucher, les odeurs, les couleurs. Par exemple, la synesthésie

Je sentis

La dièse des oranges

Aux catalogues des odeurs

devient

I felt

The sharps of oranges

chez le traducteur et

I smelled

Sharps of oranges

chez Barbara Godard. "La saison des groseilles", " the Gooseberry Season " chez Alexandre Amprimoz se transforme en "Red Currant Time " chez la traductrice.

Le corps sexué apparaît de façon plus explicite dans les traductions par les femmes qui semblent insister sur l'aspect duratif du plaisir. Le dernier vers du poème de Cécile Cloutier

L'émail parfumé

Des châteaux de plumes

Acheva l'effort de ta caresse

est rendu par le traducteur par " ended the effort of your caress", ce qui chez la traductrice devient " completed the effort of your hand". Pour prendre un dernier exemple de cette présence du corps sexué,

L'émail parfumé

Des châteaux de plumes

Acheva l'effort de ta caresse.

The scented enamel

of feather castles

Ended the effort of your caress.

\section{A. Amprimoz}

The perfumed enamet

of feather castles

Completed the effort of your hand.

B. Godard

15. A Clash of Symbols (1979) : (la Nef des sorcières), translated by Linda Gaboriau, Toronto, Coach House Press.

16. Sylvia Plath (1976) : Trois Femmes, traduit par Laure Vernière et Owen Leeming, Paris. dans le texte de Nicole Brossard, l'écrivain déclare "ce soir j'entre dans l'histoire sans relever ma jupe $" 17$; traduction de David Ellis " without pulling up my skirt "18, traduction de Linda Gaboriau " without opening my legs". Cette traduction concrète, brutale par Linda Gaboriau fait partie d'une coloration générale de violence des textes féministes, violence souvent exprimée en termes et métaphores sexuels et que le traducteur ne rend pas toujours avec la même force. Il apparaît que la plupart des variations présentées par les différentes traductions sont plus ou moins directement fonction de la connaissance ou de la méconnaissance par le traducteur ou la traductrice de l'"intertexte féministe". Si le traducteur ignore quels sont les thèmes principaux des textes féministes, à savoir la sexualité et son polymorphisme, les fonctions reproductrices, l'accouchement, le viol, le sang, s'il ne sait que les femmes expérimentent avec une écriture où les mots sont les prolongements du corps, une écriture où le corps de la femme se dit, s'écrit, explore ses images, parle de son corps à corps avec la matière et avec les autres, sa traduction risque de perdre toute une coloration paralinguistique. Connaissant les positions des féministes vis-à-vis de la sexualité, David Ellis n'aurait pas traduit la phrase du texte de Nicole Brossard "les mots affluent autour du clitoris $" 19$ par " the words flow by the Clitoris". Linda Gaboriau elle a choisi comme traduction "words gather around the clitoris".

Peut-on vraiment affirmer que les différentes traductions d'un même texte poétique varient en fonction du sexe du traducteur? Une certaine interprétation psychanalytique qui relie l'acte de traduire au complexe d'OEdipe et selon laquelle la traduction est la sublimation chez le traducteur ou la traductrice du désir incestueux pour le texte mère permettrait de répondre de façon affirmative à la question posée. Nous n'aurons pas recours à la psychanalyse pour répondre à notre question. Nous dirons que, d'une part, le corpus de séquences poétiques traduites est trop réduit pour parler de différences au niveau de la syntaxe par exemple. D'autre part, les textes étudiés offrent plusieurs exemples de traductions très différentes de métaphores liées au physiologique, en particulier aux excrétions et au sexe de la femme. Chez un traducteur la "perte blanche" de la femme devient the "white loss" au lieu de "discharge 20 et la mort dans le flot menstruel de l'ovule non fécondé, mort dont nous parle Sylvia Plath dans son poème "Three Women ", s'entoure dans le texte du traducteur d'une ambiguïté sémantique ${ }^{21}$.

17. Nicole Brossard (1976) : in la Nef des sorcières, Montréal, Quinze, p. 74 .

18. David Ellis (1976) : in Exile, vol. 4, no 1, Toronto, University of Toronto Press, p. 114.

19. Ibid., p. 117.

20. Traduction proposée dans une première version en anglais du texte "Simulation " de Nicole Brossard. Cf. The Story So Far 6 edited by Nicole Brossard (1980), Toronto, Coache House Press.

21. Sylvia Plath, "Three Women ", traduit par Sinclair Robinson et Laure Vernière 


\section{LA TRADUCTION DE LA SOPHONISBE DE TRISSINO PAR MELLIN DE SAINT-GELAIS}

En 1515, devant le pape Léon X, on récitait la Sophonisbe de Trissino, première tragédie classique de la Renaissance italienne ; elle fut reprise ensuite par les histrions de Venise qui divisèrent le texte continu original en scènes et en cinq actes, réduisant également le nombre des personnages.

En 1554, au château de Blois, pour les noces du marquis d'Elbeuf, la pièce est représentée dans une traduction française de Mellin de Saint-Gelais avec la collaboration probable de François Habert.

La traduction semble fidèle; dans la plus grande partie du texte elle est presque mot à mot.

Mais les écarts isolés de sens, les tournures syntaxiques, parfois certaines transformations lorsqu'elles s'éloignent du texte de départ sont orientés par une mentalité différente des auteurs et aussi par les contraintes que le goût du public fait peser sur la représentation. C'est ainsi que des études de traduction peuvent être fécondes dans les recherches de littérature comparée.

La traduction de Saint-Gelais a des pudeurs que le texte italien n'a pas. Dans ce dernier, Sophonisbe est louée pour sa beauté ; on fait ostensiblement son éloge. Le texte français est plus discret et omet les passages trop élogieux pour accorder plus de relief à la valeur morale de l'héroïne.

Non far Signor del ciel, non far servire A gente iniqua une belta si rara (p. 8)

est traduit

O Dieu! ne permettez pas que ce malheur advienne

et de bonté si rare et vertu te souvienne ( $p$. 169)

22. Cf. François Peraldi, "Pour traduire ' Un coup de dés..." ", in Meta (1978) : vol. 23, no 1, pp. 109-110.
Beauté est remplacé par bonté et vertu : toute la perspective sur la protagoniste change. Ailleurs Bella donna (p. 16) est traduit par aimable personne ( $p$. 180). Un passage italien qui dit "elle lava à l'eau du fleuve ses beaux membres "(p. 49) est supprimé dans le texte français. Fréquemment des expressions se rapportant au corps sont omises dans la traduction telles : "in bocca d'une donna" (p. 18), "il suo bel volto" (p. 63). Mellin va jusqu'à supprimer des compliments galants faits aux suivantes "Vero ornamento a la Citta di Cirta" (p. 8). Un passage où Sophonisbe qui va mourir fait l'éloge de son lit nuptial a dû paraître inconvenant et ne se trouve pas dans le texte français

\section{O Letto mio \\ Ove deposi il fior de la mia vita \\ Rimanti in pace (p. 52)}

Ces omissions et changements transforment l'atmosphère de la pièce. Elles sont à sens unique. Doit-on les attribuer à Saint-Gelais lui-même ou aux exigences du public?

Un autre changement important est la modification de l'arrière-plan religieux par souci de rectifications historiques.

Trissino prête à Sophonisbe et à Massinissa un comportement religieux quasi chrétien. SaintGelais modifie cette situation. Dio (p. 37) ou Iddio (p. 55) est remplacé par les dieux (pp. 203, 228). Le texte italien emploie les expressions Non piaccia a Dio (p. 4) et La sentenzia del ciel (p. 5) qui sont remplacées en français par le mot fortune (p. 167) ou bien par volonté divine. Quand on insiste trop sur l'intervention divine, le français omet simplement le passage.

Cette transformation va parfois jusqu'au changement d'adjectif : santa luce (p. 19) est remplacé par invisible lumière (p. 184).

Certains changements sont provoqués par le souci de vraisemblance historique. Massinissa dans les deux textes prépare son mariage au temple, mais dans le texte traduit, il n'y a pas de prêtre (sacerdote), il n'y a pas de " oui "rituel.

Un passage joue sur l'équivoque. Les deux textes emploient l'expression Reine du ciel au moment où Sophonisbe va faire une offrande, à Dieu dans le texte italien, à Junon dans le texte français.

Les transformations les plus importantes sont stylistiques. La pièce italienne est en hendécasyllabes non rimés (sciolti); la pièce française en prose sauf les choeurs dont les mètres sont variables. Au lyrisme italien s'oppose le ton dominé et raisonneur du texte français, à la simplicité directe s'oppose la complication des phrases françaises.

Comparons ces deux textes

Ove si puo fuggir? che luogo abbiamo,

Che si conservi, e che da lor ci asconda. Se l'ajuto divin non ci difende? (p. 11) 
En quel lieu de sûreté nous saurions nous retirer, qui nous puisse défendre d'eux si Dieu seul ne nous conserve? (p. 175)

Une longue phrase remplace deux interrogations, la répétition de che interrogatif ou relatif intensifie l'expression de l'angoisse.

Même les récits historiques dont la traduction est assez littérale diffèrent par des Donc des de sorte que (pp. 8, 172) ou par des tournures hypotaxiques. Souvent des juxtapositions avec des particules de liaison en italien deviennent des propositions subordonnées en français.

Parfois les répétitions qui intensifient l'émotion en italien sont omises en français.

Dove, dove n'andrai, dove mi lasci? (p. 10)

est traduit simplement par

suis-je demeurée (p. 173)

$\grave{A}$ chaque page on pourrait relever les traits qui refroidissent le texte français. $O$ Padre ! $O$ caro Padre! (p. 10) est rendu par Ô Asdrubal! ô cher père! (p. 174). Des interjections en style direct sont reprises en style indirect (pp. 12,173). La vivacité de l'italien est ainsi perdue. Des expressions comme $O$ dura sorte ! (p. 48), O cara libertate (p. 22), si importantes pour l'action dramatique, disparaissent dans le texte français.

Des mots sont traduits par des équivalents moins violents, arroganza (p. 33) par braveté (p. 199), superbo (p. 34) par avantageux, felicita (p. 23) par prospérité (p. 191), dolore par incommodité (p. 167) et une angoscioso ... dolore (p. 35) devient une déconvenue (p. 201). L'intensité psychologique en souffre : la litote française détruit le naturel italien. Même si ces mots français avaient alors un sens plus fort qu'aujourd'hui, on doit reconnaître l'orientation française vers une expression plus retenue.

Nous reparlerons des suppressions, mais les ciseaux ont souvent choisi les passages les plus lyriques.

Parfois - le cas n'est pas fréquent - la mise en sourdine de l'émotion est due à la suppression d'images. À un moment, Sophonisbe se compare à un navire désarmé sur une mer houleuse. Cette comparaison est omise. La phrase italienne

Le soavi parole e i dolci prieghi

Farian le Tigre divenir pietose (p. 14)

est platement remplacée par

Vos doulces paroles et prières méritent trouver

non seulement pitié mais faveur (p. 178)

Sur le plan linguistique ce changement de ton est sans doute la caractéristique principale de cette "trahison" de Mellin. Mais il y a des transforma- tions structurales souvent dans la même ligne, qui modifient le jeu scénique.

Un point capital est la différence de rang social des personnages. Bien que la Sophonisbe habitât un palazzo, celui-ci est parfois appelé simplement casa, ce que Saint-Gelais traduit par château. Une simple coupe (coppa) devient un vase d'or. La pièce française double le nombre des serviteurs et ceux-ci sont anoblis. Un famiglio se multiplie en un premier et un second gentilhomme, une serva devient des femmes première, seconde et troisième de la reine. Une simple amie Erminia est devenue une dame de chambre. Le cadre français possède donc plus de grandeur et de noblesse.

Mais c'est le dernier acte qui subit la plus importante transformation scénique.

Dans le texte italien, après un dialogue pathétique avec son amie Erminia, Sophonisbe qui a bu le poison offert par Massinissa, expire sur la scène. Dans la pièce française Sophonisbe se retire dans sa chambre, puis le Choeur chante sa mort dont le récit à Massinissa est fait sur la scène par les dames de compagnie. Ce changement est conforme - presque prématurément - aux " convenances", il crée une distance qui fait manquer à la pièce l'objectif que s'était fixé Trissino dans sa présentation à Léon $\mathrm{X}$, "de provoquer la compassion ". L'effet cathartique est amoindri.

Certaines autres modifications furent apportées au texte pour des raisons esthétiques. De longs morceaux de bravoure furent coupés en deux par une réplique ; parfois un dialogue est condensé en une réplique pour écourter la pièce. La tendance française est à l'abréviation.

Si des passages du texte italien ont été supprimés, par contre le texte francais contient quelques ajouts. Examinons la nature de ces transformations.

Mellin de Saint-Gelais ne croit pas opportun de traduire entièrement le long texte du début qui est une mise en situation historique ; le récit des circonstances préparatoires au drame est abrégé. Il ne se complaît pas non plus aux nombreux éloges de Sophonisbe et à tous les compliments flatteurs qui, dans la pièce italienne, valorisent l'héroïne et rendent son drame plus pathétique.

Un écart cependant est surprenant et nous ne pouvons l'expliquer : à deux endroits, des passages de deux vers seulement sont omis; tous les deux ont le même sujet, la promesse de Massinissa de ne pas livrer Sophonisbe aux Romains ; or cette promesse est un élément essentiel de l'action.

Par contre, le texte français ajoute parfois quelques mots à litalien surtout dans la seconde moitié de l'oeuvre.

Il y a deux espèces d'ajouts français ; des précisions sont parfois données sur l'action surtout dans de longues répliques fragmentées en français; la seconde espèce est composée de sententieuses généralités morales. Elles semblent montrer que l'auteur français avait peut-être moins le souci du drame luimême et plus celui de la leçon qu'on pouvait en tirer. 
Mais toutes les remarques que je viens de faire - hormis les stylistiques - ne couvrent qu'une faible partie du texte : cependant, elles le modifient profondément.

Maintenant commence le travail du comparatiste ; les écarts repérés doivent être expliqués, resitués dans le contexte socio-culturel. L'appel du public, les conditions sociologiques de la représentation, les théories en vogue sur le théâtre, ont-ils influencé la traduction? Serait-ce aussi la personnalité du traducteur ou les limites de sa compétence? La vérification de ces hypothèses nous fournira des perspectives fécondes sur la vie littéraire du $\mathrm{XVI}^{\mathrm{e}}$ siècle.

\section{RÉFÉRENCES}

La Sophonisba, Tragedia di Gio. Giorgio Trissino (1796) : dans Teatro Italiano o sia scelta di tragedia per uso delle scena, Venezia, Stefano Orlandini, t. 1.

Sophonisba dans Mellin de Saint-Gelais (1873) OEuvres complètes, t. 3, Paris, Paul Daffis.

\section{PierRe VAN RutTen}

